\title{
LA DECLARACIÓN DE LAS NACIONES UNIDAS SOBRE LOS DERECHOS DE LOS PUEBLOS INDÍGENAS: UN HITO EN EL PROCESO DE RECONOCIMIENTO DE LOS DERECHOS INDÍGENAS
}

\author{
Felipe Gómez IsA*
}

SUMARIO: 1. INTRODUCCIÓN.-2. LOS PUEBLOS INDÍGENAS ANTE EL DERECHO INTERNACIONAL: DE LA EXCLUSIÓN A LA INCLUSIÓN.-3. RECONOCIMIENTO PROGRESIVO DE LOS DERECHOS DE LOS PUEBLOS INDÍGENAS.-3.1. Principales innovaciones de la DNUDPI.-3.2. La DNUDPI y las injusticias históricas.-3.3. La DNUDPI y el Derecho internacional.-3.4. Alcance jurídico de la Declaración.-4. CONCLUSIONES.

\section{INTRODUCCIÓN}

1. Este artículo pretende analizar el interesante, aunque también contradictorio, proceso de expansión de las normas y los mecanismos que promueven y protegen los derechos de los pueblos indígenas en el ámbito internacional. Para realizar este análisis, el artículo parte de una reflexión sobre el papel que ha desempeñado el Derecho internacional en relación con los pueblos indígenas, un papel que ha evolucionado con el paso del tiempo de una situación de exclusión y dominación bajo el Derecho internacional clásico a otra de una progresiva y relativa inclusión bajo el Derecho internacional contemporáneo. A continuación, el artículo aborda el rol de la Declaración de las Naciones Unidas sobre los Derechos de los Pueblos Indígenas (DNUDPI) en relación con el reconocimiento de derechos a los pueblos indígenas. Tras analizar su alcance jurídico y político, tratamos de desentrañar los impactos que está teniendo en los ámbitos internacionales, regionales y nacionales. Este estudio acaba con unas conclusiones en las

\footnotetext{
* Profesor titular de Derecho internacional público de la Facultad de Derecho de la Universidad de Deusto e investigador del Instituto de Derechos Humanos Pedro Arrupe (felipe.gomez@deusto.es). Este artículo ha sido elaborado en el marco de la International Research Network «Justice and Indigenous Peoples, JUSTIP» (DR 16) otorgada por el Centre National de la Recherche Scientifique, CNRS, París. Todas las páginas web han sido consultadas por última vez el 28 de noviembre de 2018.
} 
que subrayo el contradictorio papel que ha jugado el Derecho internacional a lo largo de la historia en relación con los pueblos indígenas. Actualmente, el Derecho internacional se ha convertido en un aliado estratégico de los pueblos indígenas para avanzar sus luchas en favor del reconocimiento de sus derechos. Ahora bien, también debemos ser plenamente conscientes de que estos avances en el plano jurídico internacional se enfrentan a límites y desafíos muy importantes.

\section{LOS PUEBLOS INDÍGENAS ANTE EL DERECHO INTERNACIONAL: DE LA EXCLUSIÓN A LA INCLUSIÓN}

2. El Derecho internacional tradicional jugó un papel muy destacado en la dramática historia de conquista, usurpación de la soberanía de los pueblos indígenas y expropiación de sus tierras, territorios y recursos ${ }^{1}$. El sistema jurídico emergente en Europa se convirtió en un poderoso instrumento en manos de los conquistadores para dominar y subyugar a los pueblos indígenas de las Américas ${ }^{2}$. Desde su creación, dado el protagonismo de los Estados europeos en su evolución y la posición sub-alterna de los pueblos indígenas y de otros países y pueblos no occidentales, el Derecho internacional puede ser caracterizado como un discurso hegemónico y como fuente de dominación ${ }^{3}$. Al respecto, Elvira Pulitano ${ }^{4}$ se ha referido a este ordenamiento jurídico como «esencialmente eurocéntrico». Al mismo tiempo, en un movimiento un tanto contradictorio, la denominada Escuela Española del Derecho internacional ${ }^{5}$, basada en la idea aristotélico-tomista de la sociabilidad natural y de la racionalidad de los seres humanos, defendió el carácter humano y la libertad de los indios en el contexto de la conquista española de América ${ }^{6}$. Las ideas de pensadores tales como Francisco de Vitoria o Bartolomé de las Casas abrieron la puerta a la consideración de los indígenas como seres humanos plenos

\footnotetext{
1 KeAL, P., European Conquest and the Rights of Indigenous Peoples: The Moral Backwardness of International Society, Nueva York, Cambridge University Press, 2003.

2 AnAYA, J., Indigenous Peoples in International Law, Oxford, Oxford University Press, 2004; ANGHIE, A., Imperialism, Sovereignty and the Making of International Law, Cambridge, Cambridge University Press, 2004.

3 Rajagopal, B., International Law from Below. Development, Social Movements and Third World Resistance, Cambridge, Cambridge University Press, 2003.

4 Pulitano, E., «Indigenous rights and international law: an introduction», en Pulitano, E. (ed.), Indigenous Rights in the Age of the UN Declaration, Cambridge, Cambridge University Press, 2012.

5 ScotT, J. B., The Spanish origin of International Law: Francisco de Vitoria and his law of nations, Londres, Clarendon Press, 1934. Véase también Carrillo Salcedo, J. A., "Aportaciones de Francisco de Vitoria a los fundamentos filosóficos de los derechos humanos», en MANGAS, A. (ed.), La Escuela de Salamanca y el Derecho internacional en América. Del pasado al futuro, Salamanca, Asociación Española de Profesores de Derecho Internacional y Relaciones Internacionales, 1993, pp. 49-54.

${ }^{6}$ Gómez IsA, F., «The First Cry for Justice in the Americas-From Antonio de Montesinos to the Laws of Burgos (1512)", en SuKsI, M. et al. (eds.), First Fundamental Rights Documents in Europe. Commemorating 800 Years of Magna Carta, Cambridge, Intersentia, 2015, pp. 93-105. Véase una visión crítica en Clavero, B., Derecho Global. Por una historia verosímil de los derechos humanos, Madrid, Trotta, 2014.
} 
necesitados de protección ${ }^{7}$. Lamentablemente, la conquista de América no siguió en la práctica las enseñanzas de estos precursores del actual Derecho internacional de los derechos humanos.

3. Esta situación ha permanecido sin grandes cambios hasta muy recientemente, cuando el Derecho internacional contemporáneo comenzó progresivamente a modificar su aproximación a los pueblos indígenas. Resulta muy sorprendente que todavía en la primera mitad del siglo xx Occidente siguiera pensando que estaba investido de una "misión civilizadora» para salvar a los pueblos no europeos de la ignorancia y la barbarie ${ }^{8}$. Muy en línea con este enfoque, el primer tratado internacional dirigido a los pueblos indígenas aprobado por la OIT en 1957 estaba dominado por un paradigma asimilacionista bastante evidente ${ }^{9}$. Tal y como señala el art. 2.1 del Convenio sobre Poblaciones Indígenas y Tribales (Convenio 107), «incumbirá principalmente a los gobiernos desarrollar programas coordinados y sistemáticos con miras a la protección de las poblaciones en cuestión y a su integración progresiva en la vida de sus respectivos países» (la cursiva es nuestra).

4. Las décadas de los setenta y los ochenta fueron testigo de la denominada «emergencia indígena» ${ }^{10}$, y el Derecho internacional, en particular el Derecho internacional de los derechos humanos, se convirtió en un recurso discursivo muy poderoso en manos de los pueblos indígenas para articular sus demandas tanto en espacios internos como internacionales. En opinión de Rhiannon Morgan ${ }^{11}$, «el movimiento indígena global es un ejemplo de un movimiento que ha aprovechado el potencial transformador del discurso de los derechos humanos, sirviéndose de su maleabilidad para promover la evolución y el cambio de dicho discurso». Pero el discurso de los derechos humanos también tiene sus contradicciones cuando nos aproximamos a él desde la perspectiva indígena. Tenemos que admitir que la misma noción de derechos humanos tiene ciertas reminiscencias del colonialismo. Al igual que

\footnotetext{
7 CArozza, P., «From Conquest to Constitutions: Retrieving a Latin American Tradition of the Idea of Human Rights», Human Rights Quarterly, vol. 25, 2003, p. 291. Véase también MeSA, R., "Bartolomé de las Casas, Maestro contemporáneo», en MANGAS, A., op. cit., nota 5, pp. 77-82. Una visión crítica del controvertido papel desempeñado por Bartolomé de las Casas se puede encontrar en CLAVERO, B., Genocidio y Justicia. La Destrucción de las Indias, ayer y hoy, Madrid, Marcial Pons, 2002, p. 69.

8 Esta es la esencia de algunas de las disposiciones que encontramos en el Pacto de la Sociedad de Naciones (1919), el tratado constitutivo de la primera organización internacional creada tras la Primera Guerra Mundial. De acuerdo a su art. 22, que tiene que leerse en clave colonial, «a las colonias y territorios que, a raíz de la reciente guerra, han cesado de hallarse bajo la soberanía de los Estados que los gobernaban anteriormente y que son habitados por pueblos aún incapaces de regirse por sí mismos en las condiciones particularmente difíciles del mundo moderno, deberá aplicarse el principio de que el bienestar y el desarrollo de esos pueblos constituyen una misión sagrada de civilización» (la cursiva es nuestra).

9 Thornberry, P., Indigenous Peoples and Human Rights, Manchester, Manchester University Press, 2002, pp. 329-333.

10 BengoA, J., La emergencia indígena en América Latina, México, Fondo de Cultura Económica, 2000; BRYsK, A., From Tribal Village to Global Village. Indian Rights and International Relations in Latin America, Stanford, Stanford University Press, 2000.

11 Morgan, R., Transforming Law and Institution. Indigenous Peoples, the United Nations and Human Rights, Farnham, Ashgate, 2011, p. 43.
} 
el colonialismo, «el discurso de los derechos humanos contiene ciertas asunciones implícitas sobre la naturaleza de las sociedades civilizadas y las sociedades atrasadas [...]. Los conceptos de civilización y barbarie, racionalidad y pasión, pensamientos binarios fundamentales durante la época imperial, retornan en el marco de debates contemporáneos acerca de los derechos humanos y la justicia social. La práctica de los derechos humanos cuenta con la pesada carga de la interpretación colonial de la cultura ${ }^{12}$. Muy en la misma línea, Makau Mutua ${ }^{13}$ ve la manera de como los principales defensores de los derechos humanos los han interpretado "como parte del proyecto colonial formado por la cadena que vinculaba a los misioneros cristianos, a los mercaderes del capital, y a los administradores coloniales».

5. Al mismo tiempo, y como parte del proceso de empoderamiento de los propios pueblos indígenas, desde los años setenta las Naciones Unidas se han mostrado crecientemente receptivas a sus demandas ${ }^{14}$, abriendo espacios institucionales y canales para su participación ${ }^{15}$. Los pueblos indígenas han utilizado las Naciones Unidas como una plataforma de movilización para incrementar la visibilidad de su posición de marginalidad y para perseguir algunos objetivos estratégicos en términos de reconocimiento y protección ${ }^{16}$. De alguna manera, hemos sido testigos de un proceso muy prometedor de descolonización tanto del Derecho internacional como de las Naciones Unidas ${ }^{17}$. Este proceso ha permitido a los pueblos indígenas transformarse de

12 Engle Merry, S., Human Rights and Gender Violence. Translating International Law into Local Justice, Chicago, The University of Chicago Press, 2006, p. 226.

13 Mutua, M., «The Transformation of Africa. A Critique of the Rights Discourse», en Gómez Isa, F. y DE FeYter, K. (eds.), International Human Rights Law in a Global Context, Bilbao, Deusto University Press, 2009, p. 901.

14 Willemsen-Diaz, A., «How Indigenous Peoples' Rights Reached the UN», en Charters, C. y Stavenhagen, R. (eds.), Making the Declaration Work. The United Nations Declaration on the Rights of Indigenous Peoples, Copenhague, IWGIA, 2009, pp. 16-31.

15 Varios órganos fueron creados para abordar las demandas específicas de los pueblos indígenas. Así, destacamos el Grupo de Trabajo sobre Pueblos Indígenas, el Grupo de Trabajo sobre el proyecto de Declaración sobre los derechos de los pueblos indígenas, el Foro Permanente sobre las Cuestiones Indígenas, el Relator Especial sobre los derechos de los pueblos indígenas o el Mecanismo de Expertos sobre los Derechos de los Pueblos Indígenas. La Asamblea General de las Naciones Unidas también proclamó consecutivamente dos Decenios de las Naciones Unidas sobre Pueblos Indígenas (1994-2003, y 2005-2014). El Foro Permanente para las Cuestiones Indígenas ha hecho un llamamiento recientemente para la convocatoria de un Tercer Decenio. En su opinión, «en el curso de los dos Decenios hemos visto algunos progresos [...]. Sin embargo, necesitamos asegurar y reavivar el momentum para una verdadera implementación de la Declaración», A Third International Decade of the World's Indigenous Peoples, United Nations Permanent Forum on Indigenous Issues, Dalee Sambo Dorough, Chairperson, 7 de noviembre de 2014, en http://www.un.org/esa/socdev/unpfil/documents/CommunicationUNPFII\%20Chair-3decade.pdf.

16 Bellier, I. y PrÉAUD, M., «Emerging Issues in indigenous rights: transformative effects of the recognition of indigenous peoples», The International Journal of Human Rights, vol. 16, 2012, núm. 3, pp. 474-488. Véase la omnicomprensiva obra de Oliva Martínez, D., Los Pueblos Indígenas a la conquista de sus derechos, Madrid, Boletín Oficial del Estado, 2012.

17 Desde 1945, el Derecho y las instituciones internacionales han sido utilizados por Occidente (una comunidad imaginada en sí misma) para construir e imponer un conjunto de verdades racionales basadas en valores, normas y organizaciones socio-políticas de carácter necesariamente particular pero que fueron definidas como universales. Los estudios post-coloniales han demostrado que el Dere- 
meras víctimas en actores, y de objetos de protección en verdaderos sujetos de derechos, abriendo la puerta a su adquisición de algún tipo de personalidad jurídica internacional ${ }^{18}$. La culminación de este desarrollo jurídico e institucional ha venido de la mano de la creación del Foro Permanente sobre las Cuestiones Indígenas de las Naciones Unidas en el año 2000, un órgano de naturaleza única en el que los Estados y los representantes indígenas participan en pie de igualdad ${ }^{19} \mathrm{y}$, sobre todo, de la adopción de la DNUDPI en 2007. Como el Comité sobre los Derechos de los Pueblos Indígenas de la International Law Association (ILA) ha afirmado, todos estos desarrollos demuestran que «las personas y los pueblos indígenas han sido reconocidos no solo como plenos sujetos de derechos humanos individuales sino como actores colectivos con derechos distintos y con un status diferenciado bajo el Derecho internacional» ${ }^{20}$.

\section{RECONOCIMIENTO PROGRESIVO DE LOS DERECHOS DE LOS PUEBLOS INDÍGENAS}

6. Uno de los avances más significativos en el campo de los derechos humanos en las últimas décadas ha sido el reconocimiento gradual de los derechos de los pueblos indígenas tanto a nivel doméstico como en el ámbito internacional. La OIT fue la primera organización internacional en prestar atención a la situación de marginación y exclusión que sufrían los pueblos indígenas. La adopción del Convenio 169 de la OIT en 1989 en sustitución del Convenio 107 (1957) ${ }^{21}$ fue un paso muy relevante para el reconocimiento de los derechos indígenas ${ }^{22}$, pero los Estados se han mostrado reacios a su ratificación ${ }^{23}$. Teniendo en cuenta los aspectos negativos de estas dos Conven-

cho y las instituciones internacionales, entre otras muchas estructuras de poder, fueron utilizadas por el mundo occidental para mantener sus jerarquías y sus formas de dominación. Las luchas de los pueblos indígenas han pretendido des-colonizar tanto la teoría como la práctica del «complejo ideológicoinstitucional» conocido como Derecho internacional. Sobre este desafiante proceso, véase el iluminador ensayo de Pahuja, S., Decolonising international law. Development, economic growth and the politics of universality, Cambridge, Cambridge University Press, 2011.

${ }^{18}$ Sambo Dorough, D., «The Significance of the Declaration on the Rights of Indigenous Peoples and Its Future Implementation», en Charters, C. y Stavenhagen, R. (eds.), op. cit., nota 14, p. 265; MeIJKnecht, A., Towards International Personality: The Position of Minorities and Indigenous Peoples in International Law, Oxford, Intersentia, 2001.

${ }^{19}$ García-Alix, L., The Permanent Forum on Indigenous Issues, Copenhague, IWGIA, 2003.

${ }^{20}$ International LaW Association, Rights of Indigenous Peoples, Report of The Hague Conference, 2010, p. 2.

${ }_{21}$ Veintisiete Estados han ratificado esta Convención. Desde la adopción del Convenio 169, el Convenio 107 ya no está abierto a la ratificación.

22 Rodríguez-Piñero, L., Indigenous Peoples, Post-colonialism and International Law. The ILO Regime (1919-1989), Oxford, Oxford University Press, 2005.

${ }^{23}$ Hasta el 1 de octubre de 2018, tan solo veintidós países han ratificado el Convenio 169 de la OIT (Argentina, Estado Plurinacional de Bolivia, Brasil, República Centroafricana, Chile, Colombia, Costa Rica, Dinamarca, República Dominicana, Ecuador, Fiyi, Guatemala, Honduras, México, Nepal, Holanda, Nicaragua, Noruega, Paraguay, Perú, España y la República Bolivariana de Venezuela). Disponible en http://www.ilo.org/dyn/normlex/en/f?p=1000:11300:0::NO:11300:P11300_INSTRUMENT_ID:312314. 
ciones de la OIT, el movimiento indígena global estableció como prioridad la adopción de una declaración de alcance universal que proclamase sus derechos. Tras un largo proceso de negociación en el que los pueblos indígenas fueron los principales impulsores ${ }^{24}$, finalmente la DNUDPI fue aprobada el 13 de septiembre de 2007 por la Asamblea General de las Naciones Unidas con una abrumadora mayoría ${ }^{25}$.

\subsection{Principales innovaciones de la DNUDPI}

7. La DNUDPI ha incorporado todo un conjunto de nuevos derechos, contribuyendo así a expandir de manera muy significativa el catálogo de los derechos humanos. La principal innovación conceptual se refiere al reconocimiento de derechos colectivos como complementarios a los derechos individuales clásicos. Los derechos colectivos han sido ajenos al proceso de emergencia y reconocimiento de derechos humanos en el mundo jurídico occidental ${ }^{26}$. El multiculturalismo que los pueblos indígenas defienden a la hora de acercarse a los derechos humanos desafía de una manera clara, aunque eventualmente también puede contribuir a enriquecer, una concepción de derechos humanos que hasta ahora ha sido esencialmente monocultural. Tal y como ha sido reconocido y reafirmado en el Preámbulo de la DNUDPI,

«los indígenas tienen sin discriminación todos los derechos humanos reconocidos en el Derecho internacional, y que los pueblos indígenas poseen derechos colectivos que son indispensables para su existencia, bienestar y desarrollo integral como pueblos» (la cursiva es nuestra).

8. La segunda expansión de derechos acometida por la DNUDPI es la inclusión de algunos de los denominados derechos humanos de la tercera generación, también conocidos como derechos de la solidaridad. Como sabemos, estos derechos emergieron en los años ochenta enfrentando una fuerte oposición por parte de la mayoría de los países occidentales. El más relevante de los derechos de tercera generación incluidos en la DNUDPI es el derecho al desarrollo ${ }^{27}$. A la luz del art. 23 de la DNUDPI, «los pueblos indígenas tienen derecho a determinar y a elaborar prioridades y estrategias para el ejercicio de su derecho al desarrollo». En la misma línea, el art. 29.1 de la Decla-

24 Bellier, I., «Les peuples autochtones aux Nations Unies: un nouvel acteur dans la fabrique des normes internationales», Critique Internationale, vol. 54, 2012, núm. 1, pp. 61-80.

25 Torrecuadrada García-Lozano, S., «La Declaración de las Naciones Unidas sobre los derechos de los pueblos indígenas: antecedentes, consecuencias y perspectivas», Estudios Internacionales. Revista del Instituto de Estudios Internacionales de la Universidad de Chile, 2010, núm. 165, pp. 7-32.

${ }^{26}$ En cambio, en otros contextos como el africano, los derechos colectivos forman parte esencial de su concepción acerca de los derechos humanos. Véase al respecto la Carta Africana de Derechos Humanos y de los Pueblos (1981).

27 Gómez IsA, F., «The Right to Development, Translating Indigenous Voice(s) into Development Theory and Practice», en Wouters, J. et al. (eds.), The World Bank Legal Review Volume 6. Improving Delivery in Development: The Role of Voice, Social Contract, and Accountability, Washington D. C., World Bank Publications, 2015, pp. 91-102. 
ración establece que «los pueblos indígenas tienen derecho a la conservación y protección del medio ambiente y de la capacidad productiva de sus tierras o territorios y recursos».

9. Finalmente, la DNUDPI también reconoce la necesidad del consentimiento previo, libre e informado de los pueblos indígenas cuando los Estados adoptan medidas legislativas o administrativas que les pueden afectar ${ }^{28}$. Mientras que el Convenio 169 está basado en la consulta a los pueblos indígenas, la DNUDPI da un nuevo paso y exige que la consulta debe ir encaminada a la obtención de su consentimiento previo, libre e informado. Tal y como viene establecido en el art. 19 de la Declaración,

«los Estados celebrarán consultas y cooperarán de buena fe con los pueblos indígenas interesados por medio de sus instituciones representativas antes de adoptar y aplicar medidas legislativas o administrativas que los afecten, a fin de obtener su consentimiento libre, previo e informado».

\subsection{La DNUDPI y las injusticias históricas}

10. Otra gran innovación de la Declaración es el reconocimiento de las injusticias históricas como impedimento para el disfrute de los derechos humanos por parte de los pueblos indígenas. De hecho, nos encontramos ante una de las primeras referencias a esta cuestión en un instrumento jurídico internacional.

11. La adopción de la DNUDPI en 2007 refleja una de las aspiraciones más profundas de los pueblos indígenas y su confianza en el Derecho internacional y en los derechos humanos como armas poderosas para modificar patrones muy enraizados de dominación y exclusión que han afectado a los pueblos indígenas desde la época de la colonia ${ }^{29}$. La propia adopción de la Declaración se puede interpretar como un intento de reparar los errores históricos sufridos por los pueblos indígenas ${ }^{30}$. De manera muy significativa, la DNUDPI incluye una referencia explícita a las injusticias históricas. En su Preámbulo, la Asamblea General de las Naciones Unidas enfatizó que

«los pueblos indígenas han sufrido injusticias históricas como resultado, entre otras cosas, de la colonización y de haber sido desposeídos de sus tierras, territorios y recursos, lo que les ha impedido ejercer, en particular, su derecho al desarrollo de conformidad con sus propias necesidades e intereses» (la cursiva es nuestra).

\footnotetext{
28 Rombouts, S., Having a Say. Indigenous Peoples, International Law and Free, Prior and Informed Consent, Oisterwijk, Wolf Legal Publishers, 2014, p. 174.

${ }_{29}$ Glenn, P., "The Three Ironies of the UN Declaration on the Rights of Indigenous Peoples», en Allen, S. y XanthaKi, A. (eds.), Reflections on the UN Declaration on the Rights of Indigenous Peoples, Oxford, Hart Publishing, 2011, pp. 171-182.

${ }^{30}$ Gómez Isa, F., «Repairing Historical Injustices: Indigenous Peoples in Post-Conflict Scenarios», en Oré Aguilar, G. y Gómez Isa, F. (eds.), Rethinking Transitions Equality and Social Justice in Societies Emerging from Conflict, Cambridge-Amberes, Intersentia, 2011, pp. 265-300.
} 
12. Además de esta preocupación de la Asamblea General por las injusticias históricas, también podemos observar una relación causa-efecto entre la colonización y la expropiación de tierras, territorios y recursos sufrida por los pueblos indígenas a lo largo de la historia, así como la imposibilidad para ejercer su derecho al desarrollo, cuyas consecuencias todavía sufren a día de hoy los pueblos indígenas ${ }^{31}$.

13. La parte substantiva de la Declaración contiene un gran número de disposiciones sobre el derecho de los pueblos indígenas a la reparación, algunas de las cuales tienen un claro vínculo con los errores del pasado. En este sentido, el art. 11 reconoce el derecho de los pueblos indígenas a "practicar y revitalizar sus tradiciones y costumbres culturales», lo que incluye el derecho a «mantener, proteger y desarrollar las manifestaciones pasadas, presentes y futuras de sus culturas, como lugares arqueológicos e históricos, objetos, diseños, ceremonias, tecnologías, artes visuales e interpretativas y literaturas» (la cursiva es nuestra). Algunas veces, la realización de este derecho incluye un proceso de restitución de propiedades y objetos de los que los pueblos indígenas fueron privados en el pasado. Como se señala en el párr. 2 del art. 11, «los Estados proporcionarán reparación por medio de mecanismos eficaces, que podrán incluir la restitución, [...] respecto de los bienes culturales, intelectuales, religiosos y espirituales de que hayan sido privados sin su consentimiento libre, previo e informado o en violación de sus leyes, tradiciones y costumbres». Por su parte, el art. 12 se refiere a la «repatriación de objetos de culto y de restos humanos» por parte de aquellos Estados que los posean.

14. El art. 20.2 de la DNUDPI reconoce que «los pueblos indígenas desposeídos de sus medios de subsistencia y desarrollo tienen derecho a una reparación justa y equitativa». Aunque esta disposición no especifica si se refiere o no a los impactos negativos sufridos por los indígenas en el pasado, se podría argumentar que las injusticias del pasado explican en gran parte las exclusiones del presente, por lo que los pueblos indígenas deberían tener derecho a la reparación.

15. Como vemos, el derecho de los pueblos indígenas a la compensación, incluyendo algunas disposiciones clave para poder buscar la reparación por injusticias de carácter histórico, se encuentra en la primera línea de la Declaración, a diferencia de otros instrumentos internacionales para la protección de los derechos de los pueblos indígenas que hemos mencionado con anterioridad.

\subsection{La DNUDPI y el Derecho internacional}

16. En gran medida, muchas de las disposiciones que encontramos en la Declaración no crean nuevos derechos, sino que se pueden considerar

31 Concha Malo, M., «Lucha por la dignidad y los derechos humanos individuales y colectivos de los pueblos de América Latina», en Mate, R. (ed.), Responsabilidad Histórica. Preguntas del nuevo al viejo mundo, Barcelona, Anthropos, 2007, p. 321. 
como una reafirmación de normas de Derecho internacional consuetudinario ya existentes ${ }^{32}$. Como ha argumentado James Anaya ${ }^{33}$, la Declaración «refleja el consenso internacional en relación con los derechos individuales y colectivos de los pueblos indígenas». A la misma conclusión ha llegado la ILA en una reciente resolución ${ }^{34}$. De acuerdo con esta autorizada interpretación, «la DNUDPI en su conjunto no se puede considerar todavía como una afirmación de Derecho internacional consuetudinario. Sin embargo, incluye varias disposiciones clave que corresponden a obligaciones de los Estados que ya existen bajo normas internacionales de carácter consuetudinario». Entre las normas que se pueden considerar como evidencia de costumbres internacionales la ILA incluye las siguientes: el derecho de los pueblos indígenas a la autodeterminación, a la autonomía y al autogobierno; el derecho a participar en los procesos de toma de decisiones nacionales que les puedan afectar; el derecho al consentimiento previo, libre e informado en proyectos que impacten de manera significativa en sus formas de vida; el derecho a su identidad cultural; el derecho a la tierra, territorios y recursos, incluyendo la restitución de las tierras ancestrales de las cuales han sido privados en el pasado; el derecho a establecer sus propias instituciones educativas y sus propios medios de comunicación; y el derecho a la reparación por las injusticias de las que los pueblos indígenas han sido objeto.

17. Esta postura ha sido rechazada expresamente por los cuatro relevantes Estados que votaron en contra de la DNUDPI (Estados Unidos, Canadá, Australia y Nueva Zelanda). La oposición más clara vino del Gobierno de los Estados Unidos, negando

«cualquier posibilidad de que la Declaración sea o pueda llegar a ser Derecho internacional consuetudinario [...]. Dado que la Declaración no describe la práctica de los Estados o acciones que los Estados se sientan en la obligación de considerar como obligaciones de carácter jurídico, no se puede citar como evidencia de la evolución de las normas de Derecho internacional consuetudinario» ${ }^{35}$.

18. El representante canadiense también compartía esta visión, señalando que la Declaración «no tiene efectos jurídicos en Canadá, y sus disposiciones no representan Derecho internacional consuetudinario» ${ }^{36}$. En el mismo sentido, el Gobierno australiano enfatizó que la DNUDPI «es una declaración de carácter aspiracional con fuerza política y moral pero no

32 ANAYA, J. y WIESSNER, S., «The UN Declaration on the Rights of Indigenous Peoples: Towards Re-empowement», Jurist, 3 de octubre de 2007, en http://jurist.org/forum/2007/10/un-declaration-onrights-of-indigenous.php.

33 ANAYA, J., Report of the Special Rapporteur on the situation of human rights and fundamental freedoms of indigenous people, S. James Anaya, UN Doc., A/HRC/9/9, 11 de agosto de 2008, p. 43.

34 International Law Association, Resolution núm. 5/2012, $75^{\text {th }}$ Conference of the ILA, Sofia, agosto de 2012, pp. 26-30, párr. 2.

35 UnITED STATES, "Observations of the United States with respect to the Declaration on the Rights of Indigenous Peoples: Explanation of Vote by Robert Hagen, U.S. Advisor, on the Declaration on the Rights of Indigenous Peoples, to the UN General Assembly», 13 de septiembre de 2007.

36 Doc. ONU A/61/PV.107, p. 13. 
jurídica [...], no pretende ser legalmente vinculante o ser reflejo de normas consuetudinarias» ${ }^{37}$. A la vez que reconocemos la relevancia que tiene la posición de estos Estados, debemos subrayar que «representan tan solo una minoría de Estados especialmente afectados por la Declaración» ${ }^{38}$. Parece bastante claro que la postura de estos Estados «no sería suficiente para impedir la emergencia de una norma de carácter consuetudinario» ${ }^{39}$, pero probablemente podrían ser considerados como objetores persistentes, dada su oposición al texto desde los primeros pasos de su elaboración. Aunque, afortunadamente para la causa de los pueblos indígenas, estos cuatro Estados han cambiado sus posturas oficiales respecto de la Declaración y la han aceptado, sin embargo, han reiterado en repetidas ocasiones que la DNUDPI no es ni legalmente vinculante ni una afirmación del Derecho internacional existente. Según la postura de Estados Unidos, «a pesar de que no es jurídicamente vinculante o una formulación del Derecho internacional existente», la Declaración «tiene fuerza tanto moral como política», y "expresa tanto las aspiraciones de los pueblos indígenas de todo el mundo como las de los Estados que buscan una mejora de las relaciones con los pueblos indígenas ${ }^{40}$. En un sentido muy similar, Australia declaró que «a pesar de que no es legalmente vinculante y no afecta al Derecho australiano, establece importantes principios internacionales a los que las naciones deben aspirar». A su vez, lo que resulta más importante desde un punto de vista jurídico, «las obligaciones actuales del Estado australiano bajo los tratados internacionales de derechos humanos se deben interpretar a la luz de los principios fundamentales contenidos en la Declaración» ${ }^{41}$. Independientemente de estas matizaciones jurídicas, debemos reconocer que la aceptación a posteriori de la DNUDPI por parte de estos Estados, constituye un paso sin precedentes en relación con documentos de esta naturaleza, y representa un signo claro de la creciente acogida de los derechos de los pueblos indígenas como parte integrante del régimen internacional de los derechos humanos. En este sentido, las palabras de la ministra de Asuntos Maoríes de Nueva Zelanda al momento de anunciar su apoyo a la DNUDPI son muy ilustrativas: «Nueva Zelanda añade su apoyo a la Declaración tanto como una afirmación de derechos

37 Ibid., p. 12.

38 BARELLI, M., «The Role of Soft Law in the International Legal System: The Case of the United Nations Declaration on the Rights of Indigenous Peoples», International and Comparative Law Quarterly, vol. 58, 2009, núm. 4, p. 967.

39 Meza-Lopehandía, M., «El Derecho internacional de los Derechos Humanos y los Pueblos Indígenas», en Aylwin, J. (coord.), Los Pueblos Indigenas y el Derecho, Santiago de Chile, LOM Ediciones, 2013, p. 466.

${ }^{40}$ Announcement of U.S. Support for the United Nations Declaration on the Rights of Indigenous Peoples, 16 de diciembre de 2010, en http://www.state.gov/documents/organization/184099.pdf. Una posición muy similar es la mantenida por el Gobierno de Canadá, Canada's Statement of Support on the United Nations Declaration on the Rights of Indigenous Peoples, 12 de noviembre de 2010, en http://www. aadnc-aandc.gc.caleng/1309374239861/1309374546142.

41 Statement on the United Nations Declaration on the Rights of Indigenous Peoples, Canberra, Parliament House, 3 de abril de 2009, en http://www.un.org/esa/socdev/unpfii/documents/Australia_official_ statement_endorsement_UNDRIP.pdf. 
fundamentales como una expresión de nuevas aspiraciones ampliamente secundadas ${ }^{42}$.

\subsection{Alcance jurídico de la Declaración}

19. A la hora de discernir el valor jurídico de la DNUDPI, se aprecian tres elementos que resultan de una extrema importancia: las circunstancias de adopción del instrumento, la precisión y naturaleza normativa de su contenido, y la existencia o no de mecanismos de implementación.

20. La aprobación de la DNUDPI en 2007 es el resultado de más de veinticinco años de intensas y controvertidas negociaciones en las que los pueblos indígenas y sus estrechos aliados alcanzaron cotas muy altas de participación. Podemos afirmar que la manera en la que se condujo el proceso, con resultados muy positivos, podría influir eventualmente en los procesos de creación de normas en otras áreas del Derecho internacional, particularmente en el ámbito de los derechos humanos. Patrick Glenn ${ }^{43}$ sitúa la Declaración como parte de un movimiento más amplio que puede tener influencia en los modos de conformación del Derecho internacional contemporáneo. En su opinión, la DNUDPI «representaría no simplemente un uso o aplicación del Derecho internacional bajo nuevas circunstancias, sino un cambio significativo en la naturaleza y en la dirección del propio Derecho internacional». La inclusividad del proceso, y el apoyo prestado por parte de un amplio espectro de órganos del sistema de las Naciones Unidas y por parte incluso de muchos Estados, proporciona a la Declaración altos grados de legitimidad, algo que incrementará necesariamente su aceptación y cumplimiento.

21. El voto final sobre la DNUDPI en la Asamblea General de las Naciones Unidas es también un signo del consenso internacional existente sobre el documento. Como sabemos, 144 Estados votaron a favor, once se abstuvieron $^{44}$, mientras que cuatro votaron en contra. Una interesante evolución ha tenido lugar desde 2007, ya que los cuatro Estados que votaron en contra han aceptado con posterioridad la Declaración. En la misma línea, dos de los Estados que se abstuvieron, Samoa y Colombia, también han mostrado su apoyo al documento. Este abrumador apoyo a la DNUDPI muestra un claro compromiso por parte de la comunidad internacional para avanzar en la protección y garantía de los derechos consagrados en la Declaración, lo que puede ser considerado como una evidencia clara de opinio iuris.

22. El último factor clave para poder determinar el valor jurídico de la DNUDPI es la existencia de mecanismos para su implementación. La DNU-

42 Statement by Hon Dr Pita Sharples, Minister of Maori Affairs, Announcement of New Zealand's support for the Declaration on the Rights of Indigenous Peoples, 19 de abril de 2010, en http://www.7genfund. org/sites/default/files/helpful-resources/New\%20Zealand\%20Position.pdf.

43 GLENN, P., op. cit., nota 29, p. 174.

${ }_{44}$ Se trata de los siguientes países: Azerbaiyán, Bangladesh, Bután, Burundi, Colombia, Georgia, Kenia, Nigeria, la Federación Rusa, Samoa y Ucrania. 
DPI no crea mecanismos específicos de seguimiento, sino que descansa en los órganos que ya existen tanto a nivel interno como a nivel internacional. El único órgano que se menciona explícitamente en la Declaración es el Foro Permanente para las Cuestiones Indígenas, situando al mismo «en primera línea para asegurar la efectiva implementación de la Declaración» ${ }^{45}$. A la luz del art. 42 de la DNUDPI,

«las Naciones Unidas, sus órganos, incluido el Foro Permanente para las Cuestiones Indígenas, y los organismos especializados, incluso a nivel local, así como los Estados, promoverán el respeto y la plena aplicación de las disposiciones de la presente Declaración y velarán por su eficacia».

23. De acuerdo con la interpretación desarrollada por Luis RodríguezPiñero ${ }^{46}$, el principal objetivo de esta disposición es «transversalizar y operativizar» la promoción de los derechos reconocidos en la Declaración en el marco de las actividades de los distintos órganos de las Naciones Unidas, Agencias Especializadas, organizaciones regionales y Estados. Por tanto, las medidas de implementación de la DNUDPI tendrán que ser tomadas en tres niveles paralelos e interrelacionados: internacional, regional y nacional.

24. A nivel internacional, debemos reconocer que un alto número de órganos de las Naciones Unidas y sus Agencias Especializadas están utilizando cada vez más la Declaración como parámetro de referencia a la hora de interpretar las normas internacionales y sus mandatos. En primer lugar, los mecanismos creados específicamente para abordar asuntos indígenas se refieren expresamente a la DNUDPI como guía interpretativa. Dado el rol relevante de coordinación del Foro Permanente para las Cuestiones Indígenas con el objetivo de transversalizar (mainstreaming) los temas indígenas a través de todo el sistema de las Naciones Unidas ${ }^{47}$, su posición es clave para la implementación de la Declaración. En su sesión de 2008, el Foro Permanente se congratuló por la adopción de la DNUDPI y declaró que a partir de ese momento se convertiría en «su marco legal» ${ }^{48}$. Asimismo, al Relator Especial sobre la situación de los derechos humanos de los indígenas también le fue confiado el mandato de promover la DNUDPI ${ }^{49}$, y ha trabajado intensamente para ello ${ }^{50}$.

45 BARELli, M., op. cit., nota 38, p. 978.

46 Rodríguez-PiÑero, L., «La "implementación” de la Declaración: las implicaciones del artículo 42», en Álvarez, N., Oliva, D. y ZúÑIga, N. (eds.), La Declaración sobre los derechos de los pueblos indígenas. Hacia un mundo intercultural y sostenible, Madrid, Los Libros de la Catarata, 2009, pp. 74-75.

47 En 2002 se creó el Grupo Inter-Agencias de Apoyo a las Cuestiones Indígenas para ayudar al Foro Permanente en su objetivo de promover el diálogo y la cooperación entre los órganos y agencias de las Naciones Unidas que tratan temas indígenas. Este Grupo está compuesto por 31 órganos, departamentos, fondos y agencias del sistema de las Naciones Unidas, BuRgER, J., «Making the Declaration Work for Human Rights in the UN System», en CHARTERS, C. y StAVENHAGEN, R. (eds.), op. cit., nota 14, pp. 309-310.

48 Foro Permanente de las Naciones Unidas para las Cuestiones Indígenas, «Informe de la Séptima Sesión», Doc. ONU E/C.19/2008/23.

49 Consejo de Derechos Humanos, Resolución 6/12, 28 de septiembre de 2007.

50 ANAYA, J., Report of the Special Rapporteur on the situation of human rights and fundamental freedoms of indigenous people, S. James Anaya, Doc. ONU A/HRC/9/9, 11 de agosto de 2008. 
25. Finalmente, el recientemente creado Mecanismo de Expertos sobre los Derechos de los Pueblos Indígenas (MEDPI) también es un órgano que puede contribuir a la implementación de la DNUDPI. Dadas sus funciones técnicas y consultivas como un órgano compuesto por cinco expertos independientes, el MEDPI se sitúa en una "posición privilegiada para contribuir a promover interpretaciones autorizadas de los estándares establecidos en la Declaración» ${ }^{51}$.

26. Los órganos creados por los tratados de derechos humanos, los conocidos como comités, también han interpretado sus tratados constitutivos incorporando gradualmente los derechos de los pueblos indígenas ${ }^{52}$, y han comenzado a referirse a la DNUDPI como parámetro de referencia a la hora de interpretar los estándares jurídicos internacionales. En esta línea, la reciente Conferencia Mundial sobre Pueblos Indígenas invitó a los comités de derechos humanos «a que examinen la Declaración en el marco de sus mandatos respectivos», alentando a los Estados «a que incluyan [...] información sobre la situación de los derechos de los pueblos indígenas, incluidas las medidas adoptadas para alcanzar los objetivos de la Declaración en los informes que presentan a esos órganos y en el proceso del examen periódico universal» ${ }^{53}$.

27. Los comités que han sido pioneros en tratar de incorporar la preocupación por los derechos indígenas en su trabajo han sido el Comité de Derechos Humanos $(\mathrm{CDH})$ y el Comité para la Eliminación de la Discriminación Racial (CERD). El CDH ha interpretado progresivamente los derechos culturales consagrados en el art. 27 del Pacto Internacional de Derechos Civiles y Políticos, vinculándolos con la manera en como los pueblos indígenas disfrutan de sus territorios y de sus recursos naturales. Así, el comité ha observado que «la cultura se manifiesta de muchas formas, inclusive un modo particular de vida relacionado con el uso de recursos terrestres, especialmente en el caso de los pueblos indígenas. Ese derecho puede incluir actividades tradicionales tales como la pesca o la caza y el derecho a vivir en reservas protegidas por la ley» ${ }^{54}$ (la cursiva es nuestra). De manera muy similar, el CERD también ha establecido un fuerte vínculo entre la discriminación racial y la desposesión de los pueblos indígenas de sus tierras y recursos naturales. En este sentido, el comité ha afirmado que es consciente del hecho que «en muchas regiones del mundo se ha discriminado y sigue discriminándose a los pueblos indígenas, y se les ha privado de sus derechos humanos y libertades fundamentales, y concretamente, de que los colonizadores, las empresas comerciales y las empresas de Estado les han arrebatado sus tierras y sus recursos» ${ }^{55}$. Es por

51 Rodríguez-Piñero, L., "Where Appropriate: Monitoring/Implementing of Indigenous Peoples' Rights under the Declaration», en Charters, C. y Stavenhagen, R. (eds.), op. cit., nota 14, p. 334.

52 THORnBERry, P., op. cit., nota 9, p. 116.

53 Conferencia Mundial sobre los Pueblos Indígenas, Documento final de la reunión plenaria de alto nivel de la Asamblea General conocida como Conferencia Mundial sobre los Pueblos Indígenas, Doc. ONU A/RES/69/2, 22 de septiembre de 2014, párr. 29.

54 Comité de Derechos Humanos, Observación General núm. 23: Derechos de las minorías (artículo 27), 8 de abril de 1994, Doc. ONU CCPR/C/21/Rev.1/Add.5, párr. 7.

55 Comité para la Eliminación de la Discriminación Racial, Recomendación General núm. 23, Derechos de los pueblos indígenas (1997), Doc. ONU A/52/18, párr. 3. 
todo ello que el comité «exhorta especialmente a los Estados Partes a que reconozcan y protejan los derechos de los pueblos indígenas a poseer, explotar, controlar y utilizar sus tierras, territorios y recursos comunales, y en los casos en que se les ha privado de sus tierras y territorios, de los que tradicionalmente eran dueños, o se han ocupado o utilizado esas tierras y territorios sin el consentimiento libre e informado de esos pueblos, que adopten medidas para que les sean devueltos» ${ }^{56}$.

28. La Convención sobre Derechos del Niño (CDN) ha sido uno de los primeros tratados de derechos humanos que explícitamente reconoció los derechos de los menores indígenas ${ }^{57}$, y el Comité de Derechos del Niño ha tratado cada vez con mayor insistencia de promover la aplicación de la DNUDPI. Así, el comité ha instado a los Estados, en un Comentario General particular sobre los derechos de las niñas y niños indígenas, "a que adopten criterios fundados en los derechos con respecto a los niños indígenas sobre la base de [...] la Declaración de las Naciones Unidas sobre los derechos de los pueblos indígenas ${ }^{58}$.

29. Otros comités como el Comité para la Eliminación de Todas las Formas de Discriminación contra las Mujeres (Comité CEDAW) y el Comité sobre Derechos Económicos, Sociales y Culturales (Comité DESC) también están prestando una atención creciente a las preocupaciones específicas de los pueblos indígenas y están emitiendo decisiones y observaciones relevantes utilizando la DNUDPI como punto de referencia normativa. El Comité CEDAW se ha centrado en la situación especialmente marginada de las mujeres indígenas en relación con el disfrute de un importante número de derechos. Así, en relación con el derecho a la salud, el comité ha afirmado que "hay factores sociales que determinan el estado de salud de las mujeres y los hombres, y que pueden variar entre las propias mujeres. Por ello, debe prestarse especial atención a las necesidades y los derechos en materia de salud de las mujeres pertenecientes a grupos vulnerables y desfavorecidos como los de las [...] mujeres autóctonas» ${ }^{59}$. Asimismo, en las Observaciones Finales emitidas por el comité en relación con Nepal declaró que estaba «profundamente preocupado por las múltiples formas de discriminación contra los grupos desaventajados de mujeres tales como las Dalit y las mujeres indígenas» ${ }^{60}$. De manera muy similar, el Comité DESC se ha referido expresamente a las

56 Ibid., párr. 5.

57 De acuerdo con su art. 30, «en los Estados en que existan minorías étnicas, religiosas o lingüísticas o personas de origen indígena, no se negará a un niño que pertenezca a tales minorías o que sea indígena el derecho que le corresponde, en común con los demás miembros de su grupo, a tener su propia vida cultural, a profesar y practicar su propia religión, o a emplear su propio idioma».

58 Comité de los Derechos del NiÑo, Observación General núm. 11 (2009): Los niños indígenas y sus derechos en virtud de la Convención (sobre los Derechos del Niño), Doc. ONU CRC/C/CG/11, 12 de febrero de 2009, párr. 82.

59 Comité para la Eliminación de la Discriminación Contra la Mujer, Recomendación General núm. 24 (1999): Mujer y Salud (artículo 12), Doc. ONU A/54/38/Rev. 1, 5 de febrero de 1999, párr. 6.

60 Comité para la Eliminación de la Discriminación Contra la Mujer, Observaciones Finales, Nepal, Doc. ONU CEDAW/C/NPL/CO/4-5, 29 de julio de 2011, párr. 39. 
necesidades específicas y a la situación de especial vulnerabilidad que sufren los pueblos indígenas en relación con un amplio catálogo de derechos económicos, sociales y culturales. Por ejemplo, y en relación con el derecho a la alimentación, el comité ha subrayado que "son especialmente vulnerables muchos grupos de pueblos indígenas cuyo acceso a las tierras ancestrales puede verse amenazado ${ }^{61}$. La misma argumentación ha sido aplicada por el comité en relación con los derechos de los pueblos indígenas a la educación $^{62}$, a la salud ${ }^{63}$ o al agua ${ }^{64}$.

30. Esta prometedora evolución experimentada por los órganos de tratados ha dejado claro que «cuando los Estados valoren cuestiones relativas a violaciones de derechos humanos contra pueblos indígenas utilizarán la DNUDPI como el estándar interpretativo contra el cual juzgar el cumplimiento de las convenciones por parte de esos mismos Estados» ${ }^{65}$.

31. A nivel regional, tanto la Corte Interamericana de Derechos Humanos como la Comisión y la Corte Africanas de Derechos Humanos y de los Pueblos están haciendo uso de la DNUDPI como una de las bases jurídicas para fundamentar sus sentencias y decisiones. La DNUDPI, y el voto positivo por parte de Surinam en la Asamblea General de las Naciones Unidas, fueron utilizados por la Corte Interamericana de Derechos Humanos en el Caso del Pueblo Saramaka c. Surinam, un caso en el que la Corte afirmó los

61 Comité de Derechos Económicos, Sociales y Culturales, Comentario General núm. 12: El derecho a una alimentación adecuada (artículo 11), Doc. ONU E/C.12/1999/5, 12 de mayo de 1999, párr. 13.

${ }^{62}$ El comité ha enfatizado que los Estados tienen la obligación de «facilitar la aceptabilidad de la educación, adoptando medidas positivas para que la educación sea culturalmente aceptable para las minorías y las poblaciones indígenas», Comité DE Derechos Económicos, Sociales y Culturales, Comentario General núm. 13: El derecho a la educación (artículo 13), Doc. ONU E/C.12/1999/10, 8 de diciembre de 1999, párr. 50.

${ }^{63}$ El comité «considera que los pueblos indígenas tienen derecho a medidas específicas que les permitan mejorar su acceso a los servicios de salud y a las atenciones de la salud. Los servicios de salud deben ser apropiados desde el punto de vista cultural, es decir, tener en cuenta los cuidados preventivos, las prácticas curativas y las medicinas tradicionales», COMITÉ DE DERECHOS Económicos, SociaLES y Culturales, Comentario General núm. 14: El derecho al disfrute del más alto nivel posible de salud (artículo 14), Doc. ONU E/C.12/2000, 11 de agosto de 2000, párr. 27.

${ }^{64}$ «Los Estados Partes deberían garantizar un acceso suficiente al agua para la agricultura de subsistencia y para asegurar la de subsistencia de los pueblos indígenas», COMITÉ DE DERECHOs ECONÓmicos, Sociales y Culturales, Comentario General núm. 15: El derecho al agua (artículos 11-12), Doc. ONU E/C.12/2002/11, 20 de enero de 2002, párr. 7.

65 Trask, M. B., «Afterword: implementing the Declaration», en Pulitano, E., op. cit., nota 4, p. 335. El recién creado examen periódico universal (EPU) en el marco del Consejo de Derechos Humanos de las Naciones Unidas también se puede considerar como una oportunidad para transversalizar los derechos indígenas y la DNUDPI en el sistema de derechos humanos de Naciones Unidas. En este sentido, la Conferencia Mundial alentó «a los Estados miembros a que incluyan, según proceda, información sobre la situación de los derechos de los pueblos indígenas, incluidas las medidas adoptadas para alcanzar los objetivos de la Declaración [...] en el proceso del examen periódico universal», CoNFERENCIA Mundial sobre los Pueblos Indígenas, op. cit., nota 53, párr. 29. Lo cierto es que el EPU ha tenido hasta ahora un escaso impacto sobre los derechos de los pueblos indígenas, OLIVA MARTínEz, D., op. cit., nota 16, pp. 878-879. Véase el análisis sobre el primer ciclo del EPU y cómo se abordaron los derechos indígenas en Higgins, N., «Advancing the Rights of Minorities and Indigenous Peoples: Getting UN Attention Via the Universal Periodic Review», Netherlands Quarterly of Human Rights, vol. 32, 2014, núm. 4, pp. 379-407. 
derechos de los pueblos indígenas sobre sus tierras, territorios y recursos. La Corte estableció que, «cuando se trate de planes de desarrollo o de inversión a gran escala que tendrían un mayor impacto dentro del territorio» los Estados tienen la obligación «no solo de consultar [...] sino también de obtener el consentimiento libre, informado y previo de los pueblos indígenas» ${ }^{66}$. Nos encontramos ante una decisión muy significativa, ya que la Corte está reconociendo implícitamente que estos estándares son principios operativos del Derecho internacional independientemente del alcance normativo de la DNUDPI y a pesar de que el Estado involucrado en ese caso, Surinam, no es parte del Convenio 169 de la OIT. Más recientemente, la Corte Interamericana se ha vuelto a referir a la DNUDPI en el Caso del Pueblo Indígena Kichwa de Sarayaku c. Ecuador ${ }^{67}$. Esta decisión, junto con algunas otras sentencias recientes de la Corte Interamericana ${ }^{68}$, se pueden considerar como pasos muy positivos para la aplicación de la DNUDPI en América. En opinión de Loreto Ferrer, las decisiones de la Corte Interamericana constituyen «interpretaciones autorizadas de los derechos reconocidos en la Convención Americana [...] $\mathrm{y}$ en otros instrumentos jurídicos internacionales. Por tanto, tienen efectos erga omnes ${ }^{69}$. Como consecuencia de todo ello, las disposiciones de la DNUDPI deben ser utilizadas por los Estados al menos «como parámetros para interpretar sus obligaciones de derechos humanos» ${ }^{70}$.

32. La Comisión Africana de Derechos Humanos y de los Pueblos también se ha mostrado abierta a incorporar la DNUDPI dentro del marco jurídico que tiene que aplicar. Solamente dos meses después de la adopción de la DNUDPI, la Comisión Africana dio la bienvenida a dicha adopción, señalando que «la Declaración se va a convertir en una herramienta muy valiosa y en un punto de referencia muy importante para la promoción y la protección de los derechos de los pueblos indígenas en el continente africano» ${ }^{71}$. En una memorable decisión tomada en mayo de 2009, la Comisión Africana declaró que el desalojo forzoso por parte del Gobierno de Kenia del pueblo Endorois de sus tierras ancestrales cerca del lago Bogoria, sin consulta previa y sin ningún tipo de compensación, constituyó una violación de varios de los derechos incluidos en la Carta Africana de Derechos Humanos y de los

\footnotetext{
66 Corte Interamericana de Derechos Humanos, Caso del Pueblo Saramaka c. Surinam (Excepciones Preliminares, Fondo, Reparaciones y Costas), Sentencia de 28 de noviembre de 2007, Serie C, núm. 172, párr. 134.

67 Corte Interamericana de Derechos Humanos, Caso del Pueblo Indígena Kichwa de Sarayaku c. Ecuador (Fondo y Reparaciones), Sentencia de 27 de junio de 2012, Serie C, núm. 245, párrs. 160 y 166.

68 Véase una sentencia muy reciente en la que la Corte también alude a la DNUDPI para fundamentar el derecho de propiedad de los pueblos indígenas sobre sus territorios tradicionales, CORTE Interamericana de Derechos Humanos, Caso Pueblo Indígena Xucuru y sus miembros c. Brasil (Excepciones Preliminares, Fondo, Reparaciones y Costas), Sentencia de 5 de febrero de 2018, párr. 116.

${ }^{69}$ Ferrer, L., "The application of the United Nations Declaration on the Rights of Indigenous Peoples in Latin America», en Ferrer, L. y Borraz, P. (eds.), Indigenous Peoples' Human Rights in Domestic Courts, Madrid, Almáciga, 2013, p. 294.

70 Ibid.

71 African Commission on Human and Peoples' Rights, Resolution 121 (XXXXII) on the UN Declaration on the Rights of Indigenous Peoples, 28 de noviembre de 2007.
} 
Pueblos, y en la DNUDPI ${ }^{72}$. En una decisión más reciente en el Caso Ogiek, la Corte Africana de Derechos Humanos y de los Pueblos ha establecido que el art. 22 de la Carta Africana de Derechos Humanos y de los Pueblos «debería ser interpretado a la luz del art. 23 de la Declaración de las Naciones Unidas sobre los derechos de los pueblos indígenas» ${ }^{73}$. Como podemos comprobar, la Corte está utilizando la DNUDPI como criterio interpretativo a la hora de aplicar la Carta Africana.

33. A nivel nacional, los derechos de los pueblos indígenas también han sido reconocidos gradualmente tanto por los órganos legislativos como por órganos del Poder Judicial ${ }^{74}$, abriendo interesantes perspectivas para su efectiva implementación. En primer lugar, muchos países, especialmente de América Latina, han incorporado los derechos indígenas en sus constituciones ${ }^{75}$. El desarrollo más ambicioso ha tenido lugar en Bolivia ${ }^{76}$, donde se aprobó una ley ${ }^{77}$ para incorporar expresamente la DNUDPI en su ordenamiento jurídico interno. En la esfera judicial, tribunales como la Corte Constitucional de Colombia ${ }^{78}$, el Tribunal Constitucional de Perú ${ }^{79}$ o la Corte Suprema de Belice, han utilizado la DNUDPI para fundamentar algunas de sus senten-

72 Centre for Minority Rights Development (Kenya) and Minority Rights Group International on behalf of Endorois Welfare Council v. Kenya, Communication 276/2003, párr. 232. Véase un análisis detallado de esta decisión en Borraz, P., «The Endorois case: Indigenous Peoples' Rights in the African regional human rights system», en FERRER, L. y BorRAz, P. (eds.), op. cit., nota 69, pp. 211-216.

${ }^{73}$ African Commission on Human and Peoples' Rights v. Republic of Kenya, Application núm. 006/2012, Judgment, 26 de mayo de 2017, párr. 209. Más referencias a la DNUDPI las podemos encontrar en los párrs. 126, 127 y 181.

${ }^{74}$ Países que han afirmado el derecho de los pueblos indígenas a su identidad cultural, a sus tierras, territorios y recursos o a la autonomía y la participación, entre otros, son Argentina, Australia, Bangladesh, Botsuana, Brasil, Camboya, Canadá, Chile, Colombia, Costa Rica, India, Laos, Malaysia, México, Nueva Zelanda, Nicaragua, Noruega, Paraguay, Perú, Sudáfrica o Taiwán, entre muchos otros, International Law Assocation, Rights of Indigenous Peoples, Interim Report, The Hague Conference, 2010, pp. 49-50.

75 Clavero, B., «Derechos indígenas y Constituciones latinoamericanas», en Berraondo, M. y GóMEZ Isa, F. (eds.), Pueblos Indígenas y Derechos Humanos, Bilbao, Universidad de Deusto, 2006, pp. 313338.

${ }^{76}$ Las nuevas constituciones de Bolivia (2009) y Ecuador (2008) son claros ejemplos de una nueva ola del constitucionalismo conocida como constitucionalismo plurinacional. Véase, al respecto, ScHILLING-VACAFLOR, A. y KUPPE, R., «Plurinational Constitutionalism: A New Era of Indigenous-State Relations?», en Nolte, D. y Schilling-Vacaflor, A. (eds.), New Constitutionalism in Latin America: Promises and Practices, Farnham, Ashgate, 2012, pp. 347-370.

77 Ley 3760 de los derechos de los pueblos indígenas, de 7 de noviembre de 2007. Una experiencia no tan exitosa es la de Bangladesh, donde el Gobierno rechazó incorporar algunas disposiciones de la DNUDPI en la Constitución, aunque, por otro lado, el debate sobre la Declaración tuvo un impacto muy positivo en la posición de los pueblos indígenas en el espacio público, GerHARz, E., «Recognizing Indigenous People, The Bangladeshi Way: The United Nations Declaration, Transnational Activism and the Constitutional Amendment Affair of 2011», Indigenous Policy Journal, vol. XXIV, 2014, núm. 4.

78 Gómez IsA, F., «Cultural Diversity, Legal Pluralism, and Human Rights from an Indigenous Perspective: The Approach by the Colombian Constitutional Court and the Inter-American Court of Human Rights», Human Rights Quarterly, vol. 36, 2014, núm. 2, pp. 722-755.

79 La Corte Constitucional de Perú se ha referido al Convenio 169 de la OIT, a la jurisprudencia de la Corte Interamericana de Derechos Humanos y a la DNUDPI para afirmar los derechos de los pueblos indígenas a sus tierras y territorios. Véase Caso Comunidad Indígena Tres Islas, Sentencia del Tribunal Constitucional, exp. núm. 01126-2011-HC/TC, 11 de septiembre de 2012, párr. 23. 
cias. Es de destacar que la Corte Suprema de Belice emitió «la primera sentencia judicial que aplicaba la DNUDPI [...] apenas un mes después de su adopción ${ }^{80}$. La Corte Suprema se refirió específicamente al voto favorable de Belice respecto de la DNUDPI como muestra de un claro compromiso de cumplir con dicho instrumento. Tal y como señaló la Corte, «las resoluciones de la Asamblea General de las Naciones Unidas no son por lo general vinculantes. Pero cuando estas resoluciones o Declaraciones contienen principios de Derecho internacional general, no se espera que los Estados no las tengan en cuenta» ${ }^{81}$. En consecuencia, la Corte estableció que el art. 26 de la DNUDPI refleja «el consenso emergente y los principios generales de Derecho internacional relativos al derecho de los pueblos indígenas a sus tierras y recursos» ${ }^{82}$.

34. Si bien debemos dar la bienvenida a estos desarrollos jurisprudenciales en el ámbito doméstico, no podemos ignorar que «lo que inicialmente parecía una tendencia positiva hacia la materialización de [...] la DNUDPI a nivel interno, se ha convertido en hechos aislados y puntuales» ${ }^{83}$. Loreto $\mathrm{Fe}-$ rrer continúa con su llamada a la precaución, dado que «desde la adopción de la Declaración, la mayor parte de los Estados de América Latina se han mostrado reacios a aceptar su aplicación en la esfera doméstica, siendo relegada a un documento aspiracional [...]. Muchos jueces en la región no conocen la Declaración o la ven como un documento meramente aspiracional» ${ }^{84}$. Una opinión parecida es la expresada por Stephen Allen, para quien, en lugar de aportar pruebas inequívocas de práctica estatal favorable a ciertos derechos indígenas, los defensores de los derechos indígenas eligen selectivamente las pruebas más favorables a sus pretensiones, presentándolas como «práctica general de los Estados afectados [...], ignorando cualquier práctica en contrario» ${ }^{85}$.

35. En suma, como hemos podido comprobar, hay una práctica emergente cada vez más significativa, tanto a nivel internacional como a nivel regional y doméstico, que constituye una base muy sólida para poder defender que la DNUDPI se ha convertido en un parámetro de referencia a la hora de interpretar los derechos de los pueblos indígenas. Asimismo, al menos algunas de las disposiciones más relevantes de la DNUDPI ya se han convertido en normas de Derecho internacional consuetudinario, o están en proceso de conversión.

${ }^{80}$ Campbell, M. y Anaya, J., «The Case of the Maya Villages of Belize: Reversing the Trend of Government Neglect to Secure Indigenous Land Rights», Human Rights Law Review, vol. 8, 2008, núm. 2, p. 377.

${ }^{81}$ Manuel Coy, Maya Village of Conejo, Manuel Caal, Perfecto Makin, Melina Makin Claimants v. Attorney General of Belize, Minister of Natural Resources, and Environmental Defendants, Supreme Court of Belize, 18 de octubre de 2007, párr. 131.

82 Ibid.

83 FERRER, L., op. cit., nota 69, p. 292.

${ }^{84}$ Ibid.

85 Allen, S., «The UN Declaration on the Rights of Indigenous Peoples and the Limits of the International Legal Project», en Allen, S. y XANTHAKI, A. (eds.), op. cit., nota 29, pp. 233-234. 


\section{CONCLUSIONES}

36. Los pueblos indígenas han utilizado el Derecho internacional contemporáneo y las principales organizaciones internacionales como estrechos aliados en su lucha por el reconocimiento y en sus esfuerzos por tratar de poner fin a patrones históricos de subyugación, expropiación y asimilación cultural. Como parte de esta evolución, los pueblos indígenas se han convertido en sujetos de derecho ante el Derecho internacional, dejando de ser meros objetos de protección y cuidado. En este sentido, los pueblos indígenas han protagonizado un interesante proceso de expansión del catálogo existente de derechos humanos. La DNUDPI aprobada en 2007 representa la culminación de un largo y complicado viaje en el que los propios pueblos indígenas y sus representantes han sido sus principales impulsores. Independientemente del incierto alcance jurídico de la DNUDPI per se, podemos y debemos concluir que dicha Declaración se ha convertido en un indiscutible parámetro de referencia para interpretar los derechos de los pueblos indígenas. La experiencia reciente por parte de diversos órganos y agencias de las Naciones Unidas, de cortes y comisiones regionales de derechos humanos e, incluso, por parte de algunos tribunales domésticos, es un claro signo de la autoridad que tiene la Declaración y de su función interpretativa. Pero debemos reconocer que, a pesar de todos estos prometedores desarrollos, los derechos de los pueblos indígenas todavía se enfrentan a lo que Rodolfo Stavenhagen ha denominado «la brecha de implementación» ${ }^{86}$. Los pueblos indígenas continúan sufriendo situaciones de vulnerabilidad, exclusión y pobreza en la mayor parte de los países en los que viven, así como una creciente presión sobre sus tierras, territorios y recursos. Estos son los principales límites y desafíos que enfrentan los innegables avances producidos en el ámbito del reconocimiento jurídico internacional de los derechos de los pueblos indígenas. Todavía existe una gran distancia entre las proclamaciones formales en las Naciones Unidas y otras instancias internacionales, y la dura realidad a la que se enfrentan cotidianamente mujeres y hombres indígenas en los diferentes confines del planeta.

\section{RESUMEN}

LA DECLARACIÓN DE LAS NACIONES UNIDAS SOBRE LOS DERECHOS DE LOS PUEBLOS INDÍGENAS: UN HITO EN EL PROCESO DE RECONOCIMIENTO DE LOS DERECHOS INDÍGENAS

Los pueblos indígenas han vivido procesos históricos de invisibilidad y exclusión sistemática prácticamente desde la época de la conquista. La llegada de los Estados postcoloniales en América Latina, África y Asia tras los diferentes procesos de descolonización no trajo consigo una modificación sustancial de las relaciones de sometimiento y sumisión

86 StaVEnhagen, R., Informe del Relator Especial sobre la situación de los derechos humanos y las libertades fundamentales de los indígenas, Doc. ONU E/CN.4/2006/78/Add.5, 17 de enero de 2006, párr. 35. 
que sufrían los pueblos indígenas. A mediados del siglo xx, la comunidad internacional comenzó a prestar atención a la situación de marginación en la que vivían los pueblos indígenas. El principal objetivo era integrar a estos pueblos considerados atrasados y necesitados de protección. Ha sido este paradigma de la integración el que ha protagonizado la mayor parte de las interacciones con los pueblos indígenas, destacando el Convenio 107 de la organización Internacional del Trabajo (OIT), el primer tratado internacional aprobado para la protección de los pueblos indígenas en 1957. Esta situación comienza a cambiar con la adopción del Convenio 169 en 1989 y, especialmente, con la reciente aprobación de la Declaración de las Naciones Unidas sobre los Derechos de los Pueblos Indígenas (DNUDPI) en septiembre de 2007. A partir de este momento, los pueblos indígenas han comenzado un proceso progresivo de reconocimiento que les ha llevado a dejar de ser meramente objetos de protección y ser considerados como sujetos de derecho tanto a nivel interno como internacional.

Palabras clave: Derecho internacional, Naciones Unidas, pueblos indígenas, colonización, DNUDPI.

\section{ABSTRACT \\ THE UNITED NATIONS DECLARATION ON THE RIGHTS OF INDIGENOUS PEOPLES: A MILESTONE IN THE PROCESS OF RECOGNITION OF INDIGENOUS RIGHTS}

Indigenous peoples have lived through a process of invisibility and systematic exclusion practically ever since the era of conquest. The arrival of republican States in Latin America following the decolonization process did not involve a substantial change in the traditional relationship of subjection and submission endured by native peoples in the Americas. In the mid-twentieth century, the international community began to pay attention to the marginalized situation of indigenous peoples. The main objective was to integrate some peoples that were considered to be backward and in need of protection. It was within this paradigm that most of the interactions with indigenous peoples have occurred, such as the first international treaty adopted in this field, Convention No. 107 of the International Labour Organization (ILO, 1957). This situation began to change with the adoption of Convention No. 169 by the ILO in 1989, and especially with the recently adopted United Nations Declaration on the Rights of Indigenous Peoples (UNDRIP, 2007). From this point onwards, indigenous peoples have become subjects of rights under international law rather than objects of protection, thus becoming protagonists of a far-reaching process of expansion of human rights.

Keywords: International Law, United Nations, indigenous peoples, colonisation, UNDRIP. 